\section{(6) OPEN ACCESS}

\title{
The impact of match-play tennis in a hot environment on indirect markers of oxidative stress and antioxidant status
}

\author{
Wade L Knez, JP Périard
}

Athlete Health and Performance Research Centre, ASPETAR, Qatar Orthopaedic and Sports Medicine Hospital, Doha, Qatar

\section{Correspondence to} Dr Wade L Knez,

Aspetar-Qatar Orthopaedic and Sports Medicine Hospital, Athlete Health and Performance Research Centre, PO Box 29222, Doha, Qatar; wade.knez@aspetar.com

Accepted 15 January 2014

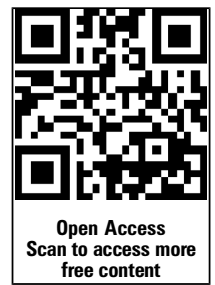

CrossMark

To cite: Knez WL, Périard JP. Br J Sports Med 2014;48:i59-i63.

\begin{abstract}
Objectives The purpose of this study was to determine the impact of changes in oxidative stress and antioxidant status in response to playing tennis in HOT $\left(\sim 36^{\circ} \mathrm{C}\right.$ and $35 \%$ relative humidity $(\mathrm{RH}))$ and $\mathrm{COOL}\left(\sim 22^{\circ} \mathrm{C}\right.$ and $70 \% \mathrm{RH})$ conditions.
\end{abstract}

Methods 10 male tennis players undertook two matches for an effective playing time (ie, ball in play) of 20 min, corresponding to $\sim 122$ and $\sim 107$ min of total play in HOT and COOL conditions, respectively. Core body temperature, body mass and indirect markers of oxidative stress (diacrons reactive oxygen metabolic test) and antioxidant status (biological antioxidant potential test) were assessed immediately prematch, midmatch and postmatch, and 24 and $48 \mathrm{~h}$ into recovery.

Results Regardless of the condition, oxidative stress remained similar throughout play and into recovery. Likewise, match-play tennis in the COOL had no impact on antioxidant status. However, antioxidants status increased significantly in the HOT compared with $\mathrm{COOL}$ environment $(p<0.05)$. Body mass losses $(\sim 0.5 \mathrm{~kg})$ were similar between conditions. Rectal temperature increased during both matches $(p<0.05)$, but with a greater magnitude in the $\mathrm{HOT}\left(39.3 \pm 0.5^{\circ} \mathrm{C}\right)$ versus $\mathrm{COOL}$ $\left(38.7 \pm 0.2^{\circ} \mathrm{C}\right)$ environment $(p<0.05)$.

Conclusions Match-play tennis in the heat does not exacerbate the development of oxidative stress, but significantly increases antioxidant status. These data suggest that the heat stress observed in the HOT environment may provide a necessary signal for the upregulation of antioxidant defence, dampening cellular damage.

\section{INTRODUCTION}

A change in oxidative balance occurs when the development of oxidative stress exceeds the antioxidant defence. An increase in oxidative stress occurs as a result of free radicals and non-radical compounds (reactive oxygen species (ROS)) modifying lipids, proteins, carbohydrates and nucleic acids. ${ }^{12}$ The sources of free radical production and subsequent oxidative stress are multifactorial, but include environmental pollution (particulate matter), ${ }^{3}$ cigarette smoke, ${ }^{4}$ physical and mental stress. ${ }^{5}$

Exercise has been shown to improve cardiovascular health, paradoxically, however, research has demonstrated that it is a significant producer of ROS caused by a leakage at the mitochondria and the increased activity of the metabolic process. ${ }^{67}$ While some research has shown that the magnitude of the exercise-induced increase in ROS is between $2-10 \%,{ }^{8}$ others have suggested that it could be as high as $10-15 \%$ above resting values. ${ }^{9}$
Notwithstanding, it is well documented that acute strenuous physical activity can cause oxidative stress, with the magnitude dependant on the type, intensity and volume of exercise. For example, oxidative stress increases with a range of exercises such as resistance work ${ }^{10}$ and endurance exercise (eg, half and full Ironman triathlons). ${ }^{11}$ Margonis et $a l^{12}$ showed in recreationally trained men, that strength training increases markers of oxidative stress (isoprostanes, the ratio of GSH/GSSG (reduced glutathione/oxidized glutathione ratio) and thiobarbituric acid reactive substances (TBARS)) via the manipulation of training volume and intensity. This result was associated with fatigue and symptoms related to over-reaching and overtraining. Although there is little research completed on elite athletes, Palazzetti et al ${ }^{13}$ investigated the impact of endurance overload training on a cohort of elite level triathletes. They too reported that overload training results in an increase in exercise-induced oxidative stress as determined by GSH/GSSG and plasma TBARS.

However, regular exercise has the capacity to enhance enzymatic antioxidant defence mechanisms that protect against and reduce the impact of oxidative stress. ${ }^{5}{ }^{14} 15$ Still, there is very little information on the impact of high-intensity intermittent acute exercise, such as tennis and football on oxidative stress, antioxidant status and the recovery process.

One study investigated the impact of intermittent exercise on markers of oxidative stress and antioxidants in elite female football players. ${ }^{16}$ The data showed that after a $90 \mathrm{~min}$ football match there was a significant increase in oxidised glutathione, but also a corresponding increase in the antioxidant defences. As such, there was no change in oxidative stress as determined by the diacrons reactive oxygen metabolic (d-ROMs) test, indicating an upregulation of antioxidants countered the increase in free radical production. However, this study did not provide information regarding changes in core temperature or the hydration status of the participants, so it is difficult to determine their influence.

Providing information regarding the hydration status can be crucial, as simply modifying the hydration status (ie, dehydration) of exercising individuals alone can result in an increase in oxidative damage. ${ }^{17}$ Previous research indicates that as dehydration increases, so too does blood viscosity and haematocrit, ${ }^{18} 19$ impacting negatively on oxygen delivery and availability. ${ }^{20}$ This has the effect of increasing the formation of free radicals, depleting antioxidant capacity ${ }^{21}$ and subsequently increasing oxidative stress. 
Interestingly, heat stress, independent of exercise has also been shown to increase oxidative stress. ${ }^{22}$ This has been proposed to occur in response to the increase in core temperature causing a disengagement of the mitochondrial respiratory chain, reducing antioxidant defences and resulting in the production of free radicals. ${ }^{23}$ The combined impact of both heat stress and exercise on the production of ROS is an important one given the potential for an additive effect. There is little research investigating these combined conditions; however, one laboratory-based study ${ }^{24}$ examined the effect of exercising at a fixed intensity $(50 \%$ of maximal oxygen consumption $\left.\left(\mathrm{VO}_{2} \mathrm{max}\right)\right)$ in a hot environment $\left(35^{\circ} \mathrm{C}, 70 \%\right.$ relative humidity $(\mathrm{RH}))$ versus exercising in a cool environment $\left(25^{\circ} \mathrm{C}, 40 \% \mathrm{RH}\right)$ on markers of oxidative stress. Data showed that while oxidative stress increased following exercise in the hot environment, it had a differential impact on markers of oxidative stress (ie, increase in $\mathrm{F}_{2}$-isoprostanes and had no impact on lipid hydroperoxides). The authors proposed that the differential outcome could be associated with the higher sensitivity of $\mathrm{F}_{2}$-isoprostanes in determining oxidative stress. However, this study did not measure oxidative stress during exercise, nor did it account for hydration status. Moreover, the impact on antioxidant status was not examined, so it is difficult to clearly establish the relationship between these two conditions.

To date, we are unaware of any studies that have sought to investigate the effect of a combination of thermal strain and high-intensity intermittent exercise on markers of oxidative stress measured by d-ROMs and antioxidants determined by biological antioxidant potential (BAP), and compared them with the time course of changes in response to exercise and recovery. Match-play tennis is a good model to investigate this impact as it is a high-intensity intermittent exercise with an overall metabolic response similar to that of prolonged moderate-intensity exercise, such as running and cycling. ${ }^{25} 26$ During match-play, work periods performed at $60-75 \%$ of $\mathrm{VO}_{2} \max$ are interspersed with periods of light activity or rest (ie, work-to-rest ratios of $1: 2$ to $1: 5){ }^{27-30}$ Therefore, the objective of the present study was to examine the impact of match-play tennis in HOT and COOL conditions in high-level players, on oxidative stress and antioxidant potential during play and into the recovery period (24 and $48 \mathrm{~h}$ postmatch). Undertaking this type of research will greatly improve our ability to better manage performance and recovery in response to intermittent exercise.

\section{MATERIALS AND METHODS \\ Participants}

Ten high-level (International Tennis Federation (ITF) rank 1-3) male tennis players volunteered to participate in this study. Participants were mean (SD) 22.6 (4.6) years of age, 182.5 (7.3) $\mathrm{cm}$ tall and weighed $79.8(7.8) \mathrm{kg}$. They had been training and competing in tennis for 17 (4) years, competed in 17 (11) tournaments per year and participated in 60 (22) matches per year. Average total training per week was 12 (6) h. On entry into the study, all participants completed medical history and exercise and lifestyle questionnaires to assess their current levels of physical activity and antioxidant supplementation. Data from the tennis players supplementing with antioxidants was excluded. Each participant was informed of the study aims, requirements and risks before providing written informed consent.

\section{Experimental protocol}

Participants played two counter-balanced simulated matches on hard-court surfaces separated by 72 or $144 \mathrm{~h}$. They were paired prior to the start of the study according to ranking and competed against the same opponent in each match. Each pair played an indoor match in a temperate environment (COOL: $21.8\left(0.1^{\circ} \mathrm{C}\right), 73.3(2.9 \% \mathrm{RH}), 19.5\left(0.3^{\circ} \mathrm{C}\right.$ wet bulb globe temperature, WBGT)) and an outdoor match in a hot environment (HOT: $36.7\left(1.6^{\circ} \mathrm{C}\right), 35.9(11.9 \% \mathrm{RH}), 33.6\left(0.9^{\circ} \mathrm{C}\right.$ WBGT)). The matches were of typical duration (COOL: 106.8 (16.9) min, HOT: 121.8 (7.9) $\mathrm{min}$ ); however, data were analysed as a function of effective playing time. More specifically, matches were separated in $2 \times 10$ min segments of effective play, which is the cumulative time spent within play, excluding the time between points and games. This approach was chosen to ensure that outcome measures were compared after an equivalent effective playing time in both conditions. Each 10 min of effective play was separated by $\sim 25 \mathrm{~min}$ to conduct body mass and athletic performance measurements. ${ }^{31}$

On arrival on match days (9:00), body mass was recorded and participants were provided with a telemetric pill (VitalSense, Mini Mitter, Respironics, Herrsching, Germany) that was inserted in the rectum to record core body temperature. A finger prick blood sample was then taken to determine prematch plasma concentrations of oxidative stress and antioxidant potential. Participants then performed a standardised warm-up of running at $9 \mathrm{~km} / \mathrm{h}$ for $5 \mathrm{~min}$ on an indoor court followed by a $10 \mathrm{~min}$ tennis-specific warm-up in the environment of play. Following the warm-up, participants undertook the match. Changes in oxidative stress and antioxidant defence, as well as body mass were determined midmatch and postmatch, as well as into recovery at 24 and $48 \mathrm{~h}$. Core body temperature was measured immediately prematch, midmatch and postmatch. The scoring and timing characteristics of the matches complied with the 2012 ITF Rules of Tennis. ${ }^{32}$

\section{Oxidative stress and antioxidant stress}

Oxidative stress was determined by assessing the blood plasma concentration of d-ROMs test. Antioxidants were evaluated by measuring the BAP test. The tests were conducted in accordance with the manufacturers instructions (Diacron International, Grossetto, Italy) using appropriate FRAS4 instrumentation (Free Radical Analytical System 4, Health \& Diagnostics Limited Co, Parma, Italy). ${ }^{33}$ Briefly, the quantification of d-ROMs is based on the concentration of modified plasma hydroperoxides $\left(\mathrm{H}_{2} \mathrm{O}_{2}\right)$, ONOO- (peroxynitrite) and $\mathrm{ROOH}$ (hydroperoxides). ${ }^{35}$ The assessment of $\mathrm{ROOH}$ is one of the simplest markers of oxidation ${ }^{36}$ and largely lipidic in nature including isoprostanes. The concentration is determined by spectrophotometry at an absorption of $505 \mathrm{~nm}$. The BAP test is based on the ability of a coloured solution, containing ferric $\left(\mathrm{Fe}^{3+}\right)$ ions bound to a special chromogenic substrate, to decolour when its $\mathrm{Fe}^{3+}$ ions are reduced to ferrous $\left(\mathrm{Fe}^{2+}\right)$ ions, as well as it can be observed by adding a reducing system (plasma antioxidant). ${ }^{37}$ The discolouration is revealed at $505 \mathrm{~nm}$ with the photometer and provides results pertaining to many non-enzymatic antioxidants including ascorbic acid, tocopherol and bilirubin. Daily calibration was carried out each morning using known standards provided by the manufacturer (Diacron International, Grossetto, Italy).

\section{Temperature and hydration measurements}

The WBGT was recorded using a QUESTemp 36 (Quest Technologies, Oconomowoc, Wisconsin, USA). During both matches participants consumed water and a commercially available sport drink (Gatorade, Chicago, Illinois, USA) ad libitum. They were also provided with bananas and granola bars (Nature Valley, General Mills, Minneapolis, Minnesota, USA). All participants kept a food diary to record the amount of food 
consumed at each meal, which were prepared for them and food including antioxidants were kept at a minimum and standardised for all players. Participants were instructed to eat and drink as they normally would during tournament play. All body mass (wearing only shorts) and sweat loss calculations were corrected for fluid consumption. On the days when players were not participating in formal testing they were still weighed and required to provide a finger prick sample to determine plasma changes in oxidative stress and antioxidants. They then followed a standardised training programme carried out by a tennis coach ( 60 $\mathrm{min})$.

\section{Statistical analysis}

All statistical calculations were performed using PASW software V.21.0 (SPSS Chicago, Illinois, USA). A repeated-measures analysis of variance was performed to test significance between and within treatments. Outcome variables were tested using Mauchly's procedure for sphericity. Whenever the data violated the assumption of sphericity, $\mathrm{p}$ values and adjusted degrees of freedom based on the Greenhouse-Geisser correction were reported instead. Where significant effects were established, pairwise differences were identified using the Bonferroni post hoc analysis procedure adjusted for multiple comparisons. The significance level was set at $\mathrm{p}<0.05$. All values are expressed as means (SD).

\section{RESULTS}

Despite fluctuations, the plasma concentration of d-ROMs did not significantly change during match-play tennis or at 24 and $48 \mathrm{~h}$ into recovery in either the HOT or COOL condition (figure 1). However, the plasma concentration of BAP was significantly higher in the HOT compared with the COOL condition $(p<0.05)$. Although, there was no significant time or interaction effect, the postmatch concentration of BAP in the HOT condition returned back to prematch concentration within $48 \mathrm{~h}$ (figure 2).

Core temperature increased over time in the COOL condition from prematch to midmatch and postmatch $(\mathrm{p}<0.05$; table 1$)$.

Core temperature in the HOT condition also showed a significant increase over the duration of the match $(\mathrm{p}<0.05)$. The magnitude of the change in core temperature from prematch to postmatch was greater in the HOT $(4 \%)$ compared with the COOL $(2.5 \%)$ conditions $(p<0.05)$. The rate of change in core

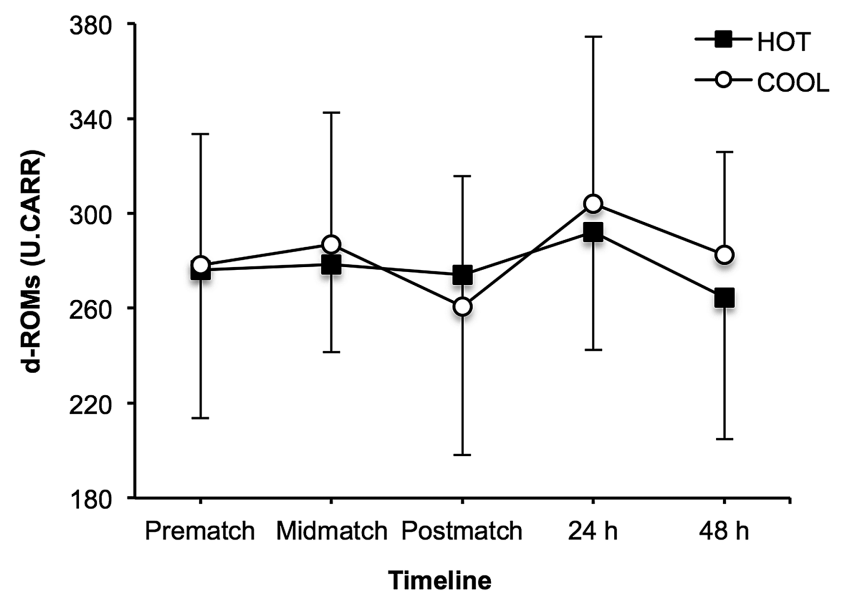

Figure 1 Changes in the diacrons reactive oxygen metabolic (d-ROMs) test during match-play tennis in COOL and HOT environments, and into the recovery period.

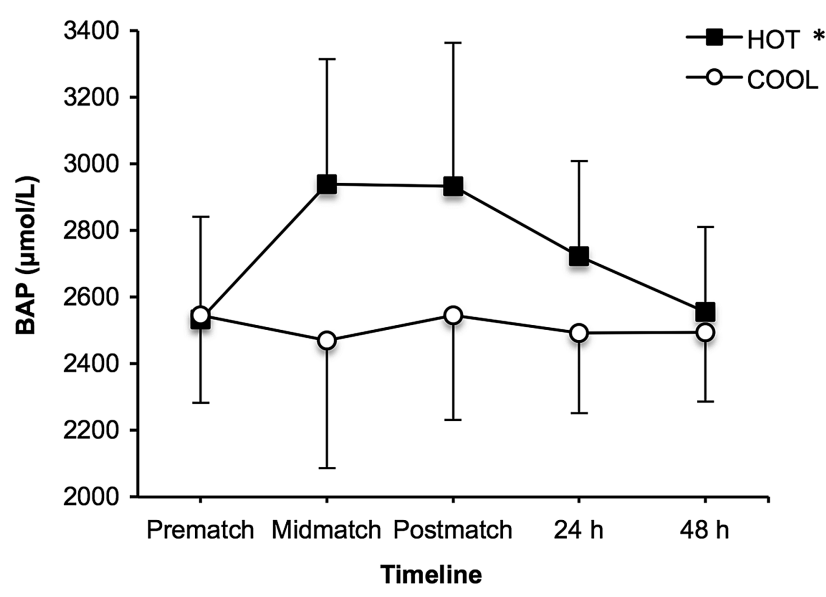

Figure 2 Changes in the biological antioxidant potential (BAP) test during match-play tennis in $\mathrm{COOL}$ and $\mathrm{HOT}$ environments, and into the recovery period. ${ }^{*}$ Significant condition impact $(p<0.05)$.

temperature in the HOT was significantly higher compared with the COOL condition (figure 3).

A greater sweat rate was observed in the HOT $(1.56 \mathrm{~L} / \mathrm{h})$ compared with the COOL $(0.96 \mathrm{~L} / \mathrm{h})$ conditions $(\mathrm{p}<0.001)$. Despite this, body mass did not change over time regardless of the environment condition (table 2). This was likely due to the greater amount of fluids consumed in the HOT $(2.02 \mathrm{~L} / \mathrm{h})$ condition in comparison to the COOL $(1.18 \mathrm{~L} / \mathrm{h})$ condition $(\mathrm{p}<0.0001)$.

\section{DISCUSSION}

The novelty of the present study lies within the characterisation of the impact of both match-play tennis (ie, high-intensity intermittent exercise) and thermal strain (ie, hot ambient conditions) on oxidative stress and antioxidant potential throughout play and into a $48 \mathrm{~h}$ recovery period. We hypothesised that the increase in core body temperature would exacerbate the exercise-induced increase in oxidative stress. However, our data did not support this proposed outcome and instead showed that regardless of the condition, match-play tennis had no significant impact of oxidative stress measured via d-ROMs. Furthermore, our data indicated that rather than the HOT condition exacerbating oxidative stress, it increased antioxidant defences. These findings suggest that there may be a critical body core temperature or rate of change in core temperature that allows for a significant upregulation in antioxidants, limiting the production of ROS and the subsequent production of oxidative stress.

Intermittent exercise in the form of match-play tennis had no impact on d-ROMs during or on recovery to $48 \mathrm{~h}$ in either the HOT or COOL condition (figure 1). However, the data showed that there was a significant difference in BAP when comparing the HOT and COOL conditions. Several studies have

Table 1 Changes in core temperature during match-play tennis in COOL and HOT environments

\begin{tabular}{|c|c|c|c|}
\hline & Prematch & Midmatch & Postmatch \\
\hline \multicolumn{4}{|c|}{ Core temperature $\left({ }^{\circ} \mathrm{C}\right)$} \\
\hline $\mathrm{COOL}$ & $37.8(0.2)$ & $38.6(0.1)^{*}$ & $38.7(0.2)^{*}$ \\
\hline НОТ & $37.8(0.3)$ & $39.1(0.4)^{*} \dagger$ & $39.3(0.5)^{*} \dagger$ \\
\hline
\end{tabular}




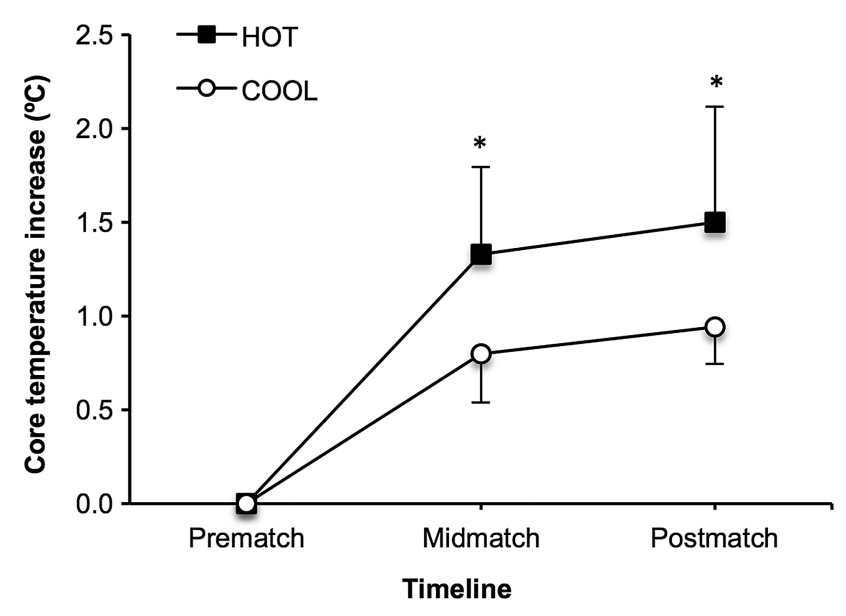

Figure 3 The effect of condition on the rate of change in core temperature. * Significant difference in the rate of change between the HOT and COOL environments $(p<0.05)$.

investigated whether acute exercise in a hot environment exacerbates the concentration of oxidative stress. $^{24} 38$ McAnulty et $a l^{24}$ obtained data from participants that completed lowintensity exercise $\left(50 \% \mathrm{VO}_{2} \max \right)$ on a treadmill in conditions of $35^{\circ} \mathrm{C}$ and $70 \% \mathrm{RH}$ until reaching a core temperature of $39.5^{\circ} \mathrm{C}$ and for an equivalent time period in temperate conditions, which resulted in a core temperature of $38.1^{\circ} \mathrm{C}$. They showed that there was an increase in oxidative stress ( $\mathrm{F}_{2}$-isoprostanes and lipid hydroperoxides) in the temperate condition, which was exacerbated by heat stress. ${ }^{24}$ Although our study does not support this finding, the study completed by McAnulty et $a l^{24}$ did not account for fluid losses and did not report changes in antioxidant concentration. In light of this, we monitored fluid intake and body mass changes throughout each match and into recovery. To simulate match and tournament conditions, we allowed participants to drink ad libitum and therefore maintain their usual drinking habits. Interestingly, we found that participants self-regulated their fluid intake such that there was no significant difference in body mass loss between the HOT and COOL condition (table 2). This is supported by the greater sweat rate and fluid intake observed in the HOT match. Noteworthy is that we also provided a commercially available carbohydrate-electrolyte solution; however, it was void of antioxidants and therefore would not have affected our results.

The present study provides support for a previous investigation completed by Laitano et $a l^{22}$ who examined the impact of exercising in the heat on the alteration in GSH/GSSG as a marker for oxidative stress. While controlling for the impact of dehydration, they showed that the combination of heat and exercise caused an upregulation of the antioxidant glutathione. Importantly however, is that there was also an increase in

Table 2 Changes in body mass during match-play tennis in COOL and HOT environments, and into the recovery period

\begin{tabular}{|c|c|c|c|c|c|}
\hline & Prematch & Midmatch & Postmatch & $24 \mathrm{~h}$ & $48 \mathrm{~h}$ \\
\hline \multicolumn{6}{|c|}{ Body mass (kg) } \\
\hline $\mathrm{COOL}$ & $82.7(9.7)$ & $82.5(9.7)$ & $82.4(9.9)$ & $82.3(9.3)$ & $82.5(9.5)$ \\
\hline HОТ & $82.3(9.6)$ & $81.9(9.9)$ & $81.8(10.4)$ & $81.6(9.6)$ & $82.0(9.5)$ \\
\hline
\end{tabular}

oxidised glutathione, but the magnitude of the change in these markers meant that there was no change in oxidative stress. Our data also indicated an upregulation of antioxidants as a result of exercising in the HOT condition compared with the COOL (figure 2). However, there was no alteration in oxidative stress. This may indicate the importance of core temperature on the impact on antioxidant defence and subsequent impact on oxidative stress. Certainly, in relation to our study, regardless of the condition, core temperature exceeded that which is considered hyperthermic ${ }^{39}$ (COOL: $38.7^{\circ} \mathrm{C}$ and HOT: $39.3^{\circ} \mathrm{C}$ ) in comparison with Laitano et $a l^{22}$ where participants reached a maximum of $38.3^{\circ} \mathrm{C}$, regardless of the condition.

As noted above, previous investigations examining the relationship between heat stress and oxidative stress did not reach a core temperature considered as hyperthermic during exercise and did not report the timeline for changes in core temperature. $^{22} 24$ Therefore, although speculative, it is possible that the core temperature obtained in the present study allowed for a further uptake of antioxidant capacity, which concomitantly maintained a stable concentration of oxidative stress. Figure 3 shows the rate of change in core temperature in response to match-play tennis in both HOT and COOL conditions. Clearly, there is a divergence between the two conditions as early as midmatch. Understanding the impact of changes in core temperature on the development of oxidative stress may provide important information about oxidative/antioxidant balance. Whether there is a potential argument to be made for a critical temperature or rate of change at which alterations in the oxidative/antioxidant balance occur, relies on future research.

\section{LIMITATIONS}

The authors recognise that there are certain limitations related to the current study. Most prominent among them is a requirement for a more detailed analysis of the markers of oxidative stress and antioxidants, including heat shock proteins. This would be desirable to facilitate a more complete understanding of the mechanisms behind the changes observed, and to confirm whether indeed there is a critical temperature that upregulates antioxidants under these circumstances in this level of athlete. However, the d-ROMs and BAP tests are simple and valid assessments of oxidative stress and antioxidant potential, ${ }^{33}$ and have been used extensively in both clinical ${ }^{40-42}$ and sporting research. ${ }^{16}{ }^{43}$ Utilising these tests in the context of match-play tennis in the field in HOT and COOL conditions, we were able to build on previous research to enable a further understanding of the relationship between the impact of environmental stress in addition to exercise and the impact on oxidative balance. Finally, we determined the changes in the oxidative stress/antioxidant balance during the recovery phase in this unique population of athletes. Noteworthy, it is important to recognise that some differences between results can be explained by differences in analytical methods and assays used.

\section{CONCLUSIONS}

This study is the first to show that in the absence of dehydration (ie, maintained body mass in HOT and COOL conditions), match-play tennis has no significant impact on oxidative stress, irrespective of environmental condition. We demonstrated that while the concentration of oxidative stress was not exacerbated in response to match-play tennis in the heat, antioxidant potential was increased compared with playing in a COOL environment. These data suggest that the environmental stress in the HOT condition may provide a necessary signal for the upregulation in antioxidant defence, dampening cellular damage. 


\section{What are the new findings?}

- Provided individuals replenish sweat losses and maintain body mass via fluid intake, intermittent exercise in a thermally challenging hot environment has no significant impact on oxidative stress.

- Our data suggest a potential critical point in core temperature or rate of change in core temperature that initiates an upregulation in antioxidant potential, which offsets the rise in oxidative stress.

\section{How might it impact on clinical practice in the near} future?

- Individual fluid consumption rates for both tennis practice and play need to be established to avoid dehydration and potential oxidative damage.

- Additional antioxidant supplementation is unnecessary to reduce oxidative stress provided individuals replenish fluid losses.

Acknowledgements The authors thank all the participants for their participation in this study. They also thank the coaches, Tim Colijn and Samuel Rota, for conducting the practises during the days between matches.

Contributors The co-author completed a significant contribution to the completion of this project and manuscript that deserves co-authorship. The co-author contributed to the planning of the study, assisted the data collection phase and co-authored the present manuscript. Given the significance of these contributing aspects he is also accountable for its data and results.

Funding This project was funded by the Aspire Zone Research Foundation.

\section{Competing interests None.}

Ethics approval The study was approved by the Shafallah Medical Genetics Centre Ethical Research Committee and conformed to the current Declaration of Helsinki guidelines.

Provenance and peer review Not commissioned; externally peer reviewed.

Open Access This is an Open Access article distributed in accordance with the Creative Commons Attribution Non Commercial (CC BY-NC 3.0) license, which permits others to distribute, remix, adapt, build upon this work non-commercially, and license their derivative works on different terms, provided the original work is properly cited and the use is non-commercial. See: http://creativecommons.org/ licenses/by-nc/3.0/

\section{REFERENCES}

1 Knez WL, Coombes JS, Jenkins DG. Ultra-endurance exercise and oxidative damage. Sports Med 2006:36:429-41.

2 Sen CK. Oxidants and antioxidants in exercise. J Appl Physiol 1995;79:675-86.

3 Gurgueira SA, Lawrence J, Coull B, et al. Rapid increases in the steady-state concentration of reactive oxygen species in the lungs and heart after particulate air pollution inhalation. Environ Health Perspect 2002;110:749-55.

4 Burke A, Fitzgerald GA. Oxidative stress and smoking-induced vascular injury. Prog Cardiovasc Dis 2003;46:79-90.

5 Bloomer RJ. Effect of exercise on oxidative stress biomarkers. Adv Clin Chem 2008;46:1-50.

6 Powers SK, Jackson MJ. Exercise-induced oxidative stress: cellular mechanisms and impact on muscle force production. Physiol Rev 2008;88:1243-76.

7 Davies KJ, Quintanilha AT, Brooks GA, et al. Free radicals and tissue damage produced by exercise. Biochem Biophys Res Commun 1982;107:1198-205.

8 Boveris $A$, Chance B. The mitochondrial generation of hydrogen peroxide. General properties and effect of hyperbaric oxygen. Biochem J 1973;134:707-16.

9 Alessio HM. Exercise-induced oxidative stress. Med Sci Sports Exerc 1993:25:218-24.

10 Steinberg J, Gainnier M, Michel F, et al. The post-exercise oxidative stress is depressed by acetylsalicylic acid. Respir Physiol Neurobiol 2002:130:189-99.

11 Knez WL, Jenkins DG, Coombes IS. Oxidative Stress in Half and Full Ironman Triathletes. Med Sci Sports Exerc 2007:39:283-8.
12 Margonis K, Fatoouris IG, Jamurtas AZ, et al. Oxidative stress biomarkers responses to physical overtraining: Implications for diagnosis. Free Radic Biol Med 2007:43:901-10

13 Palazzetti S, Richard M, Favier A, et al. Overloaded training increases exercise-induced oxidative stress and damage. Can J Appl Physiol 2003:28:588-604

14 Alessio HM, Goldfarb AH. Lipid peroxidation and scavenger enzymes during exercise: adaptive response to training. J Appl Physiol 1988;64:1333-6.

15 Radák Z, Taylor AW, Ohno H, et al. Adaptation to exercise-induced oxidative stress: from muscle to brain. Exerc Immunol Rev 2001:7:90-107.

16 Andersson $H$, Karlsen A, Blomhoff $R$, et al. Plasma antioxidant responses and oxidative stress following a soccer game in elite female players. Scand J Med Sci Sports 2009;20:600-8

17 Paik I-Y, Jeong $\mathrm{M}-\mathrm{H}$, Jin $\mathrm{H}-\mathrm{E}$, et al. Fluid replacement following dehydration reduces oxidative stress during recovery. Biochem Biophys/ Res Commun 2009;383:103-7.

18 Diaw M, Samb A, Diop S, et al. Effects of hydration and water deprivation on blood viscosity during a soccer game in sickle cell trait carriers. $\mathrm{Br}$ I Sports Med 2014;84:326-31.

19 Ajmani RS, Fleg JL, Demehin AA, et al. Oxidative stress and hemorheological changes induced by acute treadmill exercise. Clin Hemorheol Microcirc 2003:28:29-40.

20 Golbidi S, Laher I. Exercise and the cardiovascular system. Cardiol Res Pract 2012;2012:210852

21 El-Sayed MS, Ali N, El-Sayed Ali Z. Haemorheology in exercise and training. Sports Med 2005:35:649-70.

22 Laitano 0 , Kalsi KK, Pook M, et al. Separate and combined effects of heat stress and exercise on circulatory markers of oxidative stress in euhydrated humans. Eur J Appl Physiol 2010;110:953-60.

23 Hass MA, Massaro D. Regulation of the synthesis of superoxide dismutase in rat lungs during oxidant and hyperthermic stresses. J Biol Chem 1988;2:776-81.

24 McAnulty SR, McAnulty L, Pascoe DD, et al. Hyperthermia increases exercise-induced oxidative stress. Int I Sports Med 2005;26:188-92.

25 Bergeron MF, Maresh CM, Kraemer WJ, et al. Tennis: a physiological profile during match play. Int I Sports Med 1991:12:474-9.

26 Hornery DJ, Farrow D, Mujika I, et al. Fatigue in tennis: Mechanisms of fatigue and effect on performance. Sports Med 2007:37:199-212.

27 Christmass M, Richmond S, Cable N. Exercise intensity and metabolic response in singles tennis. J Sports Sci 1998;16:739-47.

28 O'Donoghue P, Ingram B. A notational analysis of elite tennis strategy. I Sports Sci 2001;19:107-15.

29 Kovacs MS. Applied physiology of tennis performance. Br J Sports Med 2006; 40:381-5; discussion 386

30 Smekal G, Duvillard von SP, Rihacek C, et al. A physiological profile of tennis match play. Med Sci Sports Exerc 2001;33:999-1005.

31 Girard $\mathrm{O}$, Christian R, Racinais $\mathrm{S}$, et al. Heat stress does exacerbate tennis-induced alterations in physical performance. Br J Sports Med 2014;48:i39-44.

32 International Tennis Federation. International Tennis Federation Rules Tennis 2012. http://www.itftennis.com. http://www.itttennis.com/media/107013/107013.pdf (accessed 9 Jul 2013)

33 Cesarone MR, Belcaro G, Carratelli $\mathrm{M}$, et al. A simple test to monitor oxidative stress. Int Angiol 1999;18:127-30.

34 Alberti A, Bolognini L, Macciantelli D. The radical cation of N, N-diethyl-paraphenylendiamine: A possible indicator of oxidative stress in biological samplesSpringer. Res Chem/ 2000;26:253-61.

35 Brigelius-Flohé R, Flohé L. Basic principles and emerging concepts in the redox control of transcription factors. Antioxid Redox Signal 2011;15:2335-81.

36 Cornelli U, Belcaro G, Finco A. The oxidative stress balance measured in humans with different markers, following a single oral antioxidants supplementation or a diet poor of antioxidants. I Cosmet Dermatol Sci App/ 2011:11:64-70.

37 Martarelli D, Verdenelli MC, Scuri S, et al. Effect of a probiotic intake on oxidant and antioxidant parameters in plasma of athletes during intense exercise training. Curr Microbiol 2011;62:1689-96.

38 Morton JP, Maclaren DPM, Cable NT, et al. Elevated core and muscle temperature to levels comparable to exercise do not increase heat shock protein content of skeletal muscle of physically active men. Acta Physiol (Oxf) 2007;190:319-27

39 McDermott BP, Casa DJ, Ganio MS, et al. Acute whole-body cooling for exercise-induced hyperthermia: a systematic review. J Athl Train 2009;44:84-93.

40 Gletsu-Miller N, Hansen JM, Jones DP, et al. Loss of total and visceral adipose tissue mass predicts decreases in oxidative stress after weight-loss surgery. Obesity 2012;17:439-46

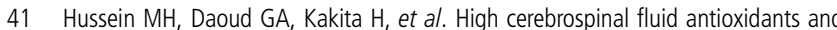
interleukin 8 are protective of hypoxic brain damage in newborns. Free Radic Res 2010:44:422-9.

42 Kotani K, Sakane N. C-reactive protein and reactive oxygen metabolites in subjects with metabolic syndrome. J Int Med Res 2012:40:1074-81.

43 Corsetti R, Villa $M$, Pasturenzi $M$, et al. Redox state in professional cyclists following competitive sports activity. Open Sports Med 2012;6:34-41. 


\section{Correction}

Knez WL, Périard JP. The impact of match-play tennis in a hot environment on indirect markers of oxidative stress and antioxidant status (Br J Sports Med 2014;48:i59-i63). The name of the second author was incorrect. It is Julien D Périard and not JP Périard.

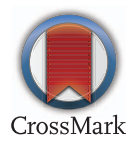

Br J Sports Med 2014;48:866. doi:10.1136/bjsports-2013-093248cor1 